American Journal of Applied Sciences 7 (8): 1118-1122, 2010

ISSN 1546-9239

(C) 2010 Science Publications

\title{
A Novel Odor Key Technique for Security Applications Using Electronic Nose System
}

\author{
Mahmoud Z. Iskandarani \\ Department of Electrical Engineering, Faculty of Engineering, \\ Al-Zaytoonah Private University of Jordan, P.O. Box 911597, \\ Post Code 11191, Amman, Jordan
}

\begin{abstract}
Problem statement: A novel approach to security applications that makes use of odors as password generators is developed and tested. The developed system detects and converts odor signals that are randomly selected to binary signals and then intelligently compares them with previously stored values. Approach: The interface device in question then either opens its interface if comparison is successful or applies security measures to extra protect resources from intrusion. The used odor keys are initially unknown to the user as it is randomly selected from a box containing many types, adding extra security to the system. This approach eliminated any possibility of hacking into the system and provides passwords which were truly secured. Results: Normal interfacing methods and algorithms were subject to hacking and infiltration. Random odor combinational keys are a novel, well secured biometric interfacing means to a wide range of systems. Design and testing of a practical electronic nose that can be used as a security interface in a wide variety of applications with a combinational odor key algorithm that ensures a hacking free environment. Conclusion/Recommendations: Successful implementation of human-machine interface through a highly secured electronic nose with very encouraging results in terms of possible odor combinations and multi-dimensional password generation.
\end{abstract}

Key words: Electronic nose, neural, signal detection, sensor arrays, computer security, biometrics

\section{INTRODUCTION}

An electronic nose is an electronic system that, just like the human nose, tries to characterize different gas mixtures using a number of individual or integrated sensors with overlapping sensitivities towards molecules. The response from a chemical sensor is usually measured as the change of some physical parameter, such conductivity voltage or current. The response times for these devices range from seconds up to a few minutes.

As a modular sensor system, it is made for detection of gases and gas mixtures, aromas and odors in an environment. It combines gas sensors for aroma detection and artificial intelligence for treatment of the measured data. This instrument is not an analytical technique but a fingerprint technique analyzing the odor as a whole. By storing the Fingerprint of the odor an electronic nose is used to recognize aromas for security control (Ampuero and Bosset, 2003; Ragazzo-Sanchez et al., 2006; Loutfi and Coradeschi, 2008).

The potential uses of nose-machines, which essentially mimic the functions of human noses but with more precision, are endless. Perfume makers are already using them to protect their patented smells against fake-fragrance; inspectors have used a high-tech nose to resolve disputes regarding freshness of fish. More exciting are the possible medical applications where scientists are researching the use of electronic noses to diagnose illness by smelling patients' breath with the possibility of installing tiny electronic noses in phone receivers, so that patients can simply breathe into the phone and wait for a diagnosis.

Researchers are investigating the use of breath analysis to identify the stages of the female menstrual cycle: the ability of electronic noses to detect ovulation could benefit both fertility treatment and birth control.

High-tech snifters may be used not just for breathsmelling but also to detect other subtle changes in body odor that can indicate disease conditions.

Our unique personal body-odor may also become an alternative form of identification, signaling the end of credit-card fraud, forgotten or misappropriated PIN numbers and fake ID cards. Companies may soon be able to replace security entry systems involving cards and codes with a device that recognizes each employee's personal odor (Matthes et al., 2005; Acevedo et al., 2007; Haddad et al., 2007; Penn et al., 2007; Wilson and Baietto, 2009). 
In this study a new approach to security systems and biometric interfacing is introduced employing a combination of odors (human and synthetic) as keys that transforms into passwords to enable access.

\section{MATERIALS AND METHODS}

The system comprises a combinations of tuned sensors (Fig. 1) coupled with sophisticated information processing. Each odorant or volatile compound presented to the sensor array produces a signature or characteristic pattern of the odorant.

By presenting many different odorants to the sensor array, a database of signatures is built up. This database of odorant signatures is then used to build odor recognition system. The goal of this process is to configure the recognition system to produce unique classifications or clustering's of each odorant so that an automated identification can be implemented.

When the sensor array is exposed to odor mixtures, containing the molecules to which the devices are sensitive, different response patterns will be created. By detecting odor patterns the nose system would then be able to classify a vapor mixture and perform security actions as required. Different levels of security exist, depending number of odors required as keys to generate a specific password (El Barbri et al., 2008; Xu et al., 2008; Qu et al., 2009; Baietto et al., 2010).

The overall system is shown in Fig. 2.

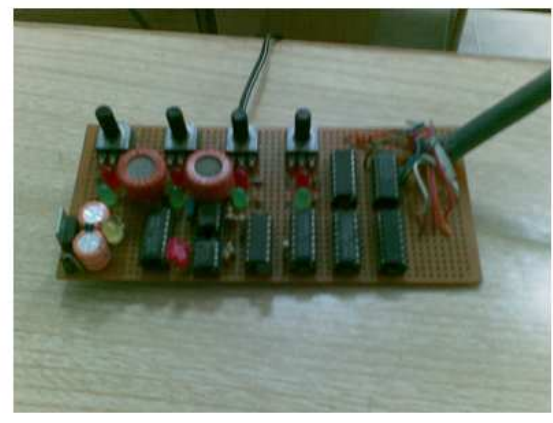

Fig. 1: Electronic nose hardware

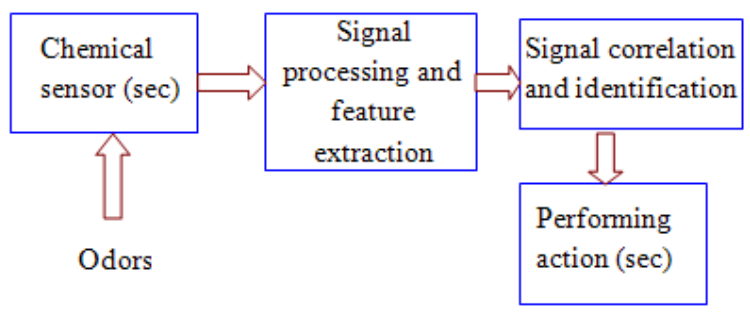

Fig. 2: Multi-sensor electronic nose system
The system main components are:

- The sensing system

- Signal processing system

- The automated Identification system

For all modes of operation, a key from a key bundle is used. The bundle and the individual keys are:

- Randomly selected (no previous knowledge of what to choose)

- Independent of other odor keys

- Have integrity whereby each odor key in the bundle has not been altered in an unauthorized manner since the time it was used

\section{RESULTS}

Table 1 shows a time related response for a 4-odor key system. For a controlled amount of odor vapor released into the security system, the time response per that amount is $v(t)$ with final steady state value reached at $\mathrm{v}(\mathrm{t}+\tau)$ as shown in Fig. 3, which also shows a comparison of time response signals.

Table 1: 4-odor keys time response

\begin{tabular}{|c|c|c|c|c|}
\hline \multirow{3}{*}{$\begin{array}{l}\text { System response- } \\
\text { recovery time (sec) }\end{array}$} & \multicolumn{4}{|c|}{ Key value (volts) } \\
\hline & Odor & Odor & Odor & Odor \\
\hline & key 1 & key 2 & key 3 & key 4 \\
\hline 0 & 2.0 & 2.0 & 2.0 & 2.0 \\
\hline 1 & 2.1 & 2.8 & 2.5 & 3.0 \\
\hline 2 & 2.8 & 5.5 & 3.5 & 4.5 \\
\hline 3 & 2.8 & 5.5 & 6.0 & 7.2 \\
\hline 4 & 2.1 & 5.0 & 7.2 & 7.2 \\
\hline 5 & 2.0 & 3.7 & 7.2 & 7.2 \\
\hline 6 & & 3.0 & 7.2 & 7.2 \\
\hline 7 & & 2.7 & 7.2 & 7.2 \\
\hline 8 & & 2.4 & 7.0 & 7.2 \\
\hline 9 & & 2.0 & 5.0 & 7.2 \\
\hline 10 & & & 4.0 & 7.2 \\
\hline 11 & & & 3.4 & 7.2 \\
\hline 12 & & & 3.2 & 7.2 \\
\hline 13 & & & 3.0 & 5.0 \\
\hline 14 & & & 2.8 & 3.8 \\
\hline 15 & & & 2.5 & 3.4 \\
\hline 16 & & & 2.3 & 3.2 \\
\hline 17 & & & 2.1 & 3.1 \\
\hline 18 & & & 2.0 & 3.0 \\
\hline 19 & & & & 2.8 \\
\hline 20 & & & & 2.7 \\
\hline 21 & & & & 2.6 \\
\hline 22 & & & & 2.5 \\
\hline 23 & & & & 2.4 \\
\hline 24 & & & & 2.2 \\
\hline 25 & & & & 2.0 \\
\hline
\end{tabular}

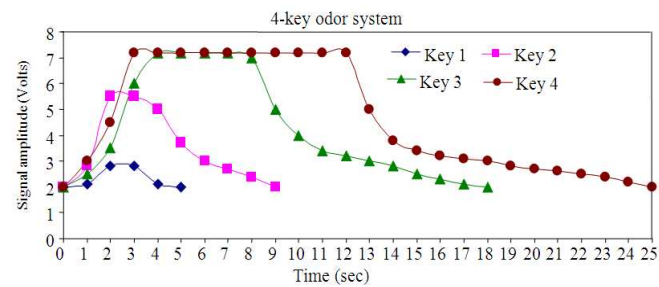

Fig. 3: Comparison between 4-odor keys time response 


\section{DISCUSION}

Each combination of signals is represented by a small set of numbers called a feature set. Using the feature set instead of the original signal allows for fast and compact data analysis. The obtained data is modeled using a simple physical description of the measurement system based on the following assumptions (Rodriguez et al., 2010; RagazzoSancheza et al., 2009; Brudzewski and Ulaczyk, 2009; Men et al., 2010; Romain and Nicolas, 2010; Bahraminejad et al., 2010; Flueckiger et al., 2009; Baha and Dibi, 2009):

- Multi-sensors, arranged in Series in the sensor chamber

- The flow of vapors through the system is unidirectional

- The vapor particles inside the chamber move with a known velocity

- The percentage of particles that diverts off the main stream is negligible

- The number of particles attached to a sensor at a particular time is proportional to the available number of particles at that time

- The response of the sensor is proportional to number of particles attached to it

The system samples the input odor and converts it into an odor key based on the condition:

$$
\mathrm{v}(\mathrm{t}+\tau)=\mathrm{v}(\mathrm{t}=0)
$$

Figure 4 show one time-based sequence 4-odor keys combination.

The system allows for tolerance, drift and saturation, hence, Eq. 1 is modified to:

$$
\mathrm{v}(\mathrm{t}+\tau)=\mathrm{v}(\mathrm{t}=0)+\Delta \mathrm{v}
$$

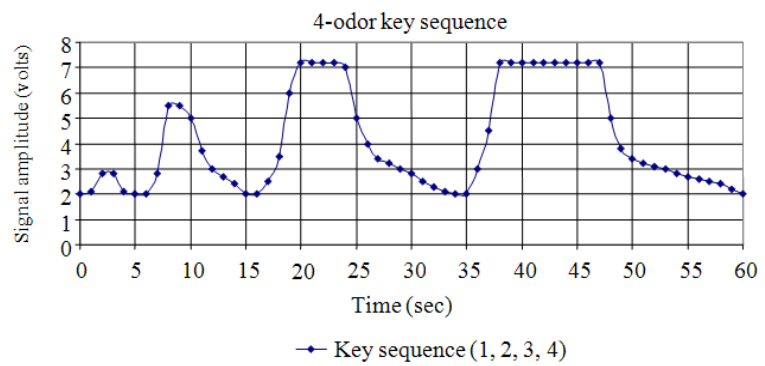

Fig. 4: Comparison between 4-odor keys time
The odor key is an accumulative of each sequence and is given by:

$$
\mathrm{ODK}_{\mathrm{i}}=\sum_{\mathrm{t}=0}^{\mathrm{t}=\mathrm{t}+\tau} \mathrm{v}_{\text {Seqi }}(\mathrm{t})
$$

To obtain an access to the system through an interface, random selection of odor keys from bundles or groups is carried out and entered, thus forming a sequence as in Eq. 4:

$$
\text { ODKSeq }=\left[\begin{array}{lll}
\mathrm{ODK}_{\mathrm{i}}, & \mathrm{ODK}_{\mathrm{j}}, \ldots, \mathrm{ODK}_{\mathrm{m}}
\end{array}\right]
$$

Each group of possibility selection has a common odor key and is placed in a matrix described by Eq. 5:

$\mathrm{ODKM}_{1}=\operatorname{prob}(\mathrm{ODKSeq})$

where the probability function randomly select odor keys from bundles that is equal to the number of time dependent sequences. Hence, for 4-key system described in this work, 4-bundles or groups are generated with 24 possible choices as shown in matrices 1-4:

$$
\text { ODKM }_{1}=\left[\begin{array}{lllr}
14 & 35 & 80 & 120 \\
14 & 80 & 35 & 120 \\
14 & 80 & 120 & 35 \\
14 & 120 & 80 & 35 \\
14 & 120 & 35 & 80 \\
14 & 35 & 120 & 80
\end{array}\right]
$$$$
\mathrm{ODKM}_{2}=\left[\begin{array}{rlrr}
35 & 14 & 80 & 120 \\
35 & 80 & 14 & 120 \\
35 & 80 & 120 & 14 \\
35 & 120 & 80 & 14 \\
35 & 120 & 14 & 80 \\
35 & 14 & 120 & 80
\end{array}\right]
$$$$
\mathrm{ODKM}_{3}=\left[\begin{array}{lllr}
80 & 14 & 35 & 120 \\
80 & 35 & 14 & 120 \\
80 & 35 & 120 & 14 \\
80 & 120 & 35 & 14 \\
80 & 120 & 14 & 35 \\
80 & 14 & 120 & 35
\end{array}\right]
$$$$
\mathrm{ODKM}_{4}=\left[\begin{array}{llll}
120 & 14 & 35 & 80 \\
120 & 35 & 14 & 80 \\
120 & 35 & 80 & 14 \\
120 & 80 & 35 & 14 \\
120 & 80 & 14 & 35 \\
120 & 14 & 80 & 35
\end{array}\right]
$$ 


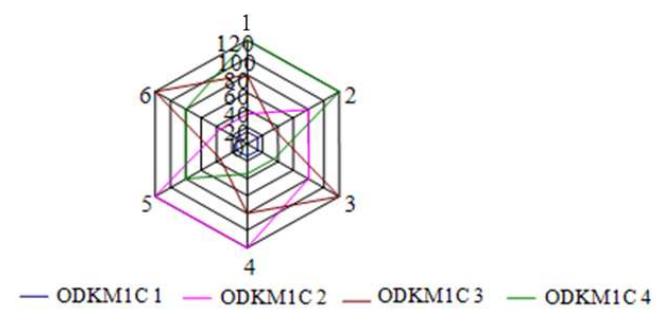

Fig. 5: Odor combinational matrix-group 1

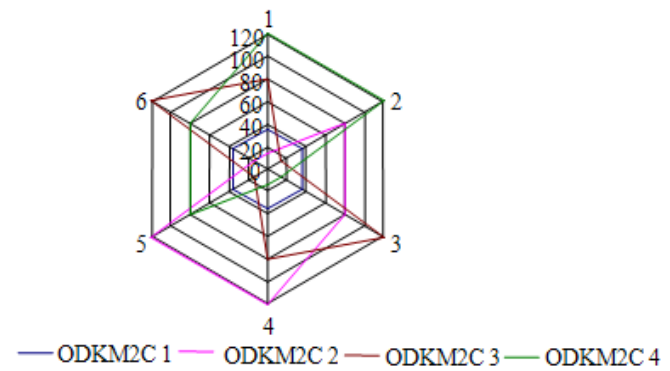

Fig. 6: Odor combinational matrix-group 2

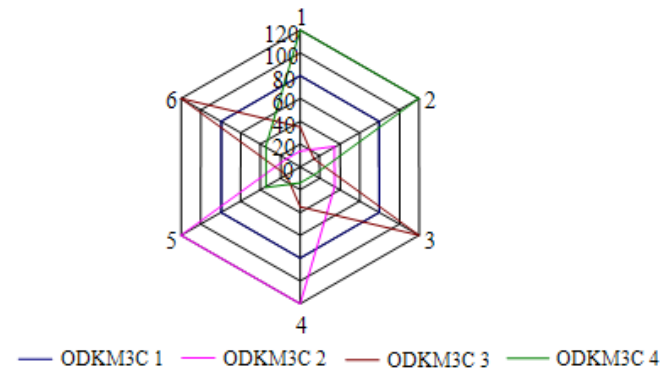

Fig. 7: Odor combinational matrix-group 3

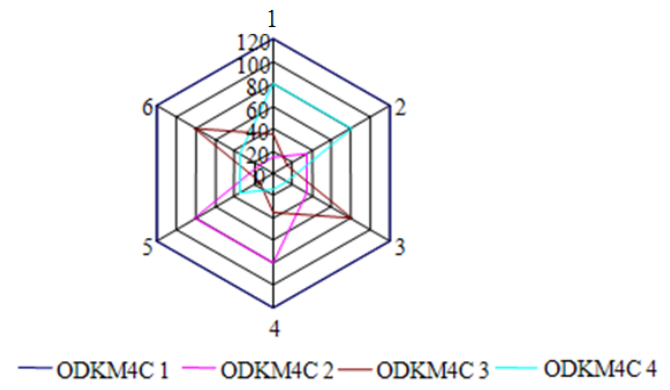

Fig. 8: Odor combinational matrix-group 4

Figure 5-8 show the four groups skeleton combinations.

Using Fig. 5-8, an algorithm operates to not only samples the practically sampled combinational odor data, but also produces other possible combinations along the connecting lines. This compensates for tolerance, sensor aging, repeatability and accuracy.

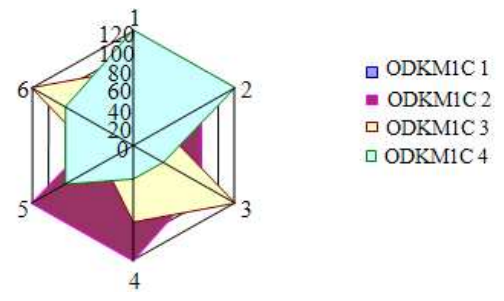

Fig. 9: Odor combinational surface-matrix group 1

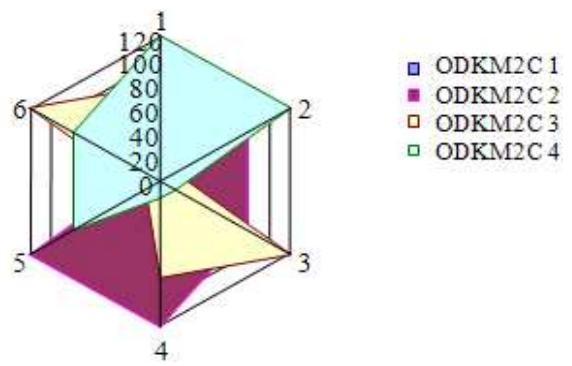

Fig. 10: Odor combinational surface-matrix group 2

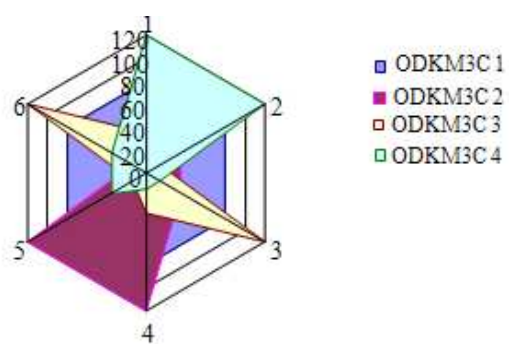

Fig. 11: Odor combinational surface-matrix group 3

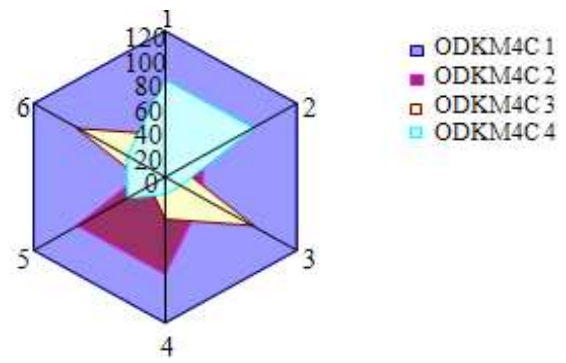

Fig. 12: Odor combinational surface-matrix group 4

Figure 9-12 showing the groups related surfaces. The surfaces show the inter-relationship between the different odor key possible combinations, with an extended algorithm used to produce indirect and twodimensional odor keys within each established odor surface.

\section{CONCLUSION}

An electronic nose system is designed and equipped with software that can detect and classify 
different odor combinations as a function of time. An array of metal oxide sensors was used for detecting vapors. The measurement circuit employs a voltage divider resistor to measure the sensitivity of each sensor. This electronic nose is controlled by specially developed software that feature extract sequences and subsequence in time domain. The system makes decisions about the odorant at certain concentrations using detection and recognition levels for the odorants. Such security system can replace traditional biometric systems like fingerprint or iris. It also replaces traditional door and safe keys with odor keys that are randomly selected and combined with impossibility of hacking.

\section{REFERENCES}

Acevedo, F.J., S. Maldonado, E. Dominguez, A. Narvaez and F. Lopez, 2007. Probabilistic support vector machines for multi-class alcohol identification. Sensors Actuast. B: Chem., 122: 227-235. DOI: 10.1016/j.snb.2006.05.033

Ampuero, S. and J.O. Bosset, 2003. The electronic nose applied to dairy products: A review. Sensors Actuat. B: Chem., 94: 1-12. DOI: 10.1016/S09254005(03)00321-6

Baha, H. and Z. Dibi, 2009. A novel neural networkbased technique for smart gas sensors operating in a dynamic environment. Sensors, 9: 8944-8960. DOI: $10.3390 / \mathrm{s} 91108944$

Bahraminejad, B., S. Basri, M. Isa and Z. Hambli, 2010. Real-time gas identification by analyzing the transient response of capillary-attached conductive gas sensor. Sensors, 10: 5359-5377. DOI: 10.3390/s 100605359

Baietto, M., A.D. Wilson, D. Bassi and F. Ferrini, 2010. Evaluation of three electronic noses for detecting incipient wood decay. Sensors, 10: 1062-1092. DOI: $10.3390 / \mathrm{s} 100201062$

Brudzewski, K. and J. Ulaczyk, 2009. An effective method for analysis of dynamic electronic nose responses. Sensors Actuat. B: Chem., 140: 43-50. DOI: 10.1016/j.snb.2009.04.028

El Barbri, N., E. Llobet, N. El Bari, X. Correig and B. Bouchikhi, 2008. Electronic nose based on metal oxide semiconductor sensors as an alternative technique for the spoilage classification of red meat. Sensors, 8: 142-156. DOI: 10.3390/s8010142

Flueckiger, J., F.K. Ko and K.C. Cheung, 2009. Microfabricated formaldehyde gas sensors. Sensors, 9: 9196-9215. DOI: 10.3390/s91109196

Haddad, R., L. Carmel and D. Harel, 2007. A feature extraction algorithm for multi-peak signals in electronic noses. Sensors Actuat. B: Chem., 120: 467-472. DOI: 10.1016/j.snb.2006.02.048
Loutfi, A. and S. Coradeschi, 2008. Odor recognition for intelligent systems. IEEE Intell. Syst., 23: 41-48. DOI: 10.1109/MIS.2008.11

Matthes, J., L. Groll and H.B. Keller, 2005. Optimal weighting of networked electronic noses for the source localization. Proceeding of the 2005 System Communication, Aug. 14-17, IEEE Computer Society, Washington DC., USA., pp: 455-460. http://portal.acm.org/citation.cfm?id=1091995

Men, H., H. Liu, L. Wang and X. Zhou, 2010. Optimization of electronic nose sensor array and its application in the classification of vinegar. Adv. Mater. Res., 121-122: 27-32. DOI: 10.4028/www.scientific.net/AMR.121-122.27

Penn, D.J., E. Gberzaucher, K. Grammer, G. Fischer and H. Soini et al., 2007. Individual and gender fingerprints in human body odor. J. R. Soc. Interface, 4: 331-340. DOI: 10.1098/rsif.2006.0182

Qu, J., Y. Chai and S.X. Yang, 2009. A real-time denoising algorithm for e-noses in a wireless sensor network. Sensors, 9: 895-908. DOI: $10.3390 / \mathrm{s} 90200895$

Ragazzo-Sanchez, J.A., P. Chalier, D. Chevalier and C. Ghommidh, 2006. Electronic nose discrimination of aroma compounds in alcoholised solutions. Sensors Actuat. B: Chem., 114: 665-673. DOI: 10.1016/j.snb.2005.05.032

Ragazzo-Sancheza, J.A., P. Chalierb, D. Chevalier-Luciab, M. Calderon-Santoyoa and C. Ghommidhc, 2009. Off-flavors detection in alcoholic beverages by electronic nose coupled to GC. Sensors Actuat. B: Chem., 140: 29-34. DOI: 10.1016/j.snb.2009.02.061

Rodriguez, J., C. Duran and A. Reyes, 2010. Electronic nose for quality control of Colombian coffee through the detection of defects in "cup tests". Sensors, 10: 36-46. DOI: 10.3390/s100100036

Romain, A.C. and J. Nicolas, 2010. Long term stability of metal oxide-based gas sensors for e-nose environmental applications: An overview. Sensors Actuat. B: Chem., 146: 502-506. DOI: 10.1016/j.snb.2009.12.027

Wilson, A.D. and M. Baietto, 2009. Applications and advances in electronic-nose technologies. Sensors, 9: 5099-5148. DOI: 10.3390/s90705099

Xu, X., F. Tian, S.X. Yang, Q. Li and J. Yan et al., 2008. A solid trap and thermal desorption system with application to a medical electronic nose. Sensors, 8: 6885-6898. DOI: 10.3390/s8116885 